\title{
Impact of smoking status on weight loss and cardiovascular risk factors
}

\author{
K Wilson, H Clark, S Hotz, R Dent
}

\section{Department of Medicine, University of Ottawa, Canada $\mathrm{K}$ Wilson \\ H Clark \\ R Dent \\ Department of Psychiatry, University of Ottawa \\ R Dent}

Loeb Research Institute, Ottawa $\mathrm{K}$ Wilson H Clark Psychology, University of Ottawa S Hotz

Correspondence to: Dr Wilson, Civic Parkdale Clinic, 737 Parkdale Avenue Suite 414, Ottawa

Hospital-Civic Site, 1053 Carling Avenue, Ottawa, (kwilson@1ri.ca)

Accepted for publication 25 October 2000
Department of Ontario, Canada K1Y 1J8

Smoking and obesity are prevalent health risks, each of which has important effects on morbidity and mortality. ${ }^{12}$ People who both smoke and are obese are at particularly high risk for cardiovascular disease and need to tackle both of these issues. Smoking cessation can be difficult to achieve in obese people because of concerns over weight gain. ${ }^{34}$ However, it is unclear whether weight loss among smokers produces the same cardiovascular benefits as in non-smokers.

In this study we attempt to: (1) determine whether smokers and non-smokers entering a weight loss programme differ with respect to cardiovascular risk factors and (2) determine whether smokers and non-smokers obtain the same level of benefit from a weight loss programme with respect to cardiovascular risk factor improvement.

\section{Methods}

The Weight Management Clinic at the Ottawa Hospital-Civic Campus offers a one year physician monitored comprehensive weight loss programme to people with high risk obesity. High risk obesity is defined as a body mass index (BMI) greater than 35 or between 30 and 35 with evidence of obesity comorbidity or presence of other cardiovascular risk factors. The programme consists of a 12 month course in lifestyle modification combined with a 13 week $900 \mathrm{kcal}$ total meal replacement (Optifast 900). Background demographic data and 13 weeks

\begin{tabular}{llllc}
\hline & Total & Smokers & Non-smokers & p Value $\neq$ \\
\hline Number & 968 & 849 & 119 & \\
Age & 44.8 & $41.3(39.5,43.2) \dagger$ & $45.3(44.6,46.0)$ & $<0.001$ \\
Men & 255 & 28 & 227 & 0.27 \\
Women & 706 & 90 & 616 & \\
Waist (inches) & 47.2 & $47.5(46.2,48.9)$ & $47.2(46.7,47.7)$ & 0.01 \\
$\Delta$ waist & 14.5 & $13.6(12.5,14.7)$ & $14.6(14.2,15.0)$ & 0.07 \\
Starting MAP (mm Hg) & 99.8 & $98.7(96.9,100.5)$ & $99.9(99.2,100.6)$ & 0.72 \\
$\Delta$ MAP & 7.41 & $7.18(5.60,8.76)$ & $7.44(6.88,7.99)$ & 0.55 \\
Starting BMI $\left(\mathrm{kg} / \mathrm{m}^{2}\right)$ & 42.8 & $42.7(41.3,44.0)$ & $42.8(42.3,43.3)$ & 0.60 \\
$\Delta$ BMI & $17.5 \%$ & $16.8(15.9,17.7)$ & $17.6(17.2,17.9)$ & 0.02 \\
Starting HDL (mmol/l) & 1.17 & $1.06(1.01,1.12)$ & $1.18(1.16,1.20)$ & $<0.001$ \\
$\Delta$ HDL & 0.9 & $4.8(1.1,8.5)$ & $0.4(-1.0,1.8)$ & 0.44 \\
Starting LDL (mmol/l) & 3.25 & $3.25(3.01,3.50)$ & $3.25(3.19,3.32)$ & 0.65 \\
$\Delta$ LDL & 23.4 & $22.1(17.3,26.9)$ & $23.6(22.1,25.1)$ & 0.11 \\
Starting triglyceride (mmol/l) & 1.80 & $1.99(1.82,2.16)$ & $1.77(1.70,1.85)$ & $<0.001$ \\
$\Delta$ triglyceride & 30.7 & $26.9(21.3,32.5)$ & $31.2(29.1,33.2)$ & 0.25 \\
Starting total cholesterol (mmol/l) & 5.20 & $5.08(4.92,5.24)$ & $5.22(5.15,5.29)$ & 0.97 \\
$\Delta$ cholesterol & 20.6 & $18.7(15.8,21.7)$ & $20.9(19.9,21.8)$ & 0.33 \\
\hline
\end{tabular}

*Smokers were those who admitted to smoking at least one cigarette/day at the time of the initial assessment. †Mean (95\% confidence intervals). ‡After adjusting for starting BMI, sex and age (stepwise regression model) where appropriate. smoking data are obtained on all patients before the first week. Weight and blood pressure are followed up weekly. Fasting serum lipid profile (total cholesterol, high density lipoprotein (HDL), low density lipoprotein (LDL) and triglyceride) is obtained at 1 and 13 weeks.

We analysed data on all patients entered into the database from September 1992 to January 2000 on whom smoking status was available. We identified smokers as those admitting to smoking one or more cigarettes at the time of their initial assessment. We determined differences in baseline characteristics and changes in BMI, waist circumference, mean arterial pressure (MAP) and cholesterol profile at 13 weeks between smokers and non-smokers using Student's $t$ tests and regression analysis ( $\chi^{2}$ tests were used to compare proportions of men and women). A linear regression model for change in BMI was developed using a forward stepwise method with a $p$ value of 0.05 for addition or removal of variables. The statistical program used for all analyses was SPSS version 8.0.

\section{Results}

The database contained smoking information on 968 people, including 119 smokers and 849 non-smokers (table 1). Sixty three per cent of those classified as smokers admitted to smoking more than 10 cigarettes/day. Smokers were significantly younger than non-smokers (41.3 and $45.3, \mathrm{p}<0.001)$, had a significantly larger initial waist size (47.5 and 47.2, $\mathrm{p}=0.01)$ and had a significantly lower HDL level (1.06 and $1.18, \mathrm{p}<0.001)$. Smokers also had a statistically significant smaller reduction in BMI $(16.8 \%$ and $17.6 \%, p=0.02)$. There was no significant difference in changes in waist circumference $(-13.6 \%$ and $-14.6 \%)$, MAP $(-7.2 \%$ and $-7.4 \%)$, HDL $(+4.8 \%$ and $+0.4 \%)$, LDL $(-22.1 \%$ and $-23.6 \%)$ or triglyceride level $(-26.9 \%$ and $-31.2 \%)$ at 13 weeks between smokers and non-smokers. Limitations of this analysis are attributable to the fact that smoking status was based on self report and that smoking status may have changed between initial assessment and week 13.

\section{Conclusion}

Smokers seem to derive the same benefit as non-smokers from a structured weight loss programme with respect to change in waist 
measurement, reduction in blood pressure and improvement in cholesterol profile. While the change in BMI between smokers and nonsmokers was statistically significant, the less than $1 \%$ difference is probably not clinically significant. The results of this study suggest that weight loss among smokers does provide benefit and need not be delayed until smoking cessation has been achieved. The decision of whether to stop smoking or lose weight first is ultimately one that should be left to the patient after consultation with their health care provider.

The authors would like to acknowledge the contributions of Rhonda Penwarden for assistance in obtaining information on the administration of the weight loss programme and Liz Yetisir for assistance in obtaining information from the database.

Funding: none.

Conflicts of interest: none.

1 Doll R, Peto R, Wheatley K, et al. Mortality in relation to smoking: 40 years' observations on male British doctors. BMF 1994;309:901-11.

2 World Health Organization. Preventing and managing the global epidemic. Report of a WHO consultation on obesity. Geneva: World Health Organization, 1998

3 Klesges RC, Meyers AW, Klesges LM, et al. Smoking, body weight, and their effects on smoking behaviour: a comprehensive review of the literature. Psychol Bull 1989;106:20430 .

4 Flegal KM, Troiano RP, Pamuk ER et al. The influence of smoking cessation on the prevalence of overweight in the United States. N Engl f Med 1995;33:1165-75. 